\title{
Wirksamkeit unabhängig von Nebenwirkungen
}

Fragestellung: Besteht ein Zusammenhang zwischen unerwünschten Arzneimittelwirkungen (UAW) und der Wirksamkeit selektiver Serotonin-Wiederaufnahmehemmer (SSRI)?

Hintergrund: Die klinische Überlegenheit von SSRI gegenüber einem Placebo ist nicht unumstritten. Nach Kirsch [1] sind SSRI einem Placebo in der antidepressiven Therapie deshalb etwas überlegen, weil die Patienten aufgrund UAW erkennen, dass sie ein Verum erhalten haben. Die dadurch induzierten höheren Erwartungen an die Wirksamkeit des Präparats würden die Placeboeffekte bei SSRI-Gabe verstärken und so zu einer Überschätzung der antidepressiven Wirksamkeit der SSRI führen. Bisher konnte nur für Fluoxetin in einer älteren Metaanalyse, in die nur sechs randomisierte, kontrollierte Studien eingeschlossen wurden, ein enger Zusammenhang zwischen UAW und SSRI-Wirksamkeit dokumentiert werden, wie er sich aus Kirschs Argumentation ergibt [2]. Eine aktuellere Metaanalyse auf Basis einer höheren Anzahl placebokon-

Barth M, Kriston L, Klostermann $\mathrm{S}$ et al. Efficacy of selective serotonin reuptake inhibitors and adverse events: metaregression and mediation analysis of placebocontrolled trials. Br J Psychiatry 2016; 208: 114-9 trollierter Studien erschien daher wünschenswert.

Patienten und Methodik: In einer umfangreichen Literaturrecherche wurden randomisierte, doppelblinde, place- bokontrollierte Studien zur Wirksamkeit einer vier- bis 13-wöchigen SSRI-Monotherapie bei Patienten mit einer akuten Episode einer mittelgradigen bis schweren Depression identifiziert. Als Wirksamkeitsmaße wurden Therapieresponse und Änderungen der Intensität depressiver Symptome gewählt, während für die Therapieverträglichkeitsbeurteilung Angaben zu UAW herangezogen wurden. Die studienübergreifende Assoziation zwischen UAW und Wirksamkeit einer SSRI-Monotherapie wurde mittels Meta-Regressionsanalysen untersucht. Eine Mediatoranalyse sollte die Frage klären, ob UAW den Zusammenhang zwischen Behandlung und Therapieresponse vermitteln.

Ergebnisse: In die Analysen der Autoren konnten insgesamt 68 klinische Studien mit 17.646 Patienten eingeschlossen werden. Die Metaanalysen ergaben, dass mit einer SSRI-Monotherapie signifikant höhere Responseraten verbunden waren als mit Placebo. Patienten aus dem SSRI-Therapiearm berichteten signifikant häufiger über UAW als Patienten aus dem Placeboarm. Es zeigte sich aber weder eine signifikante Assoziation zwischen UAW und Wirksamkeit eines SSRI, noch gab es Hinweise darauf, dass UAW die Effekte einer SSRI-Monotherapie als Mediator vermittelten.

Schlussfolgerungen: Es ist eher unwahrscheinlich, dass UAW eine Überschätzung der antidepressiven Wirksamkeit von SSRI gegenüber Placebo nach sich ziehen.

\section{- Kommentar von Roland Mergl, Leipzig}

\section{Keine klaren Belege, dass UAW die Effektivität von SSRI beeinflussen}

Es handelt sich um eine methodisch solide Übersichtsarbeit, die eine wichtige Frage zur antidepressiven Wirksamkeit der SSRI aufgreift. Sie problematisiert die Annahme, die therapeutische Überlegenheit der SSRI gegenüber einem Placebo sei allein auf den Umstand zurückzuführen, dass Patienten anhand der UAW erkannt hätten, dass sie in den Verumarm einer klinischen Studie randomisiert worden sind. Das habe wiederum höhere Erfolgserwartungen und damit einen verstärkten Placeboeffekt nach sich gezogen. Die vorliegenden Befunde legen die Existenz spezifischer antidepressiver Effekte von SSRI nahe, die über einen bloßen Placeboeffekt hinausgehen. Als kritisch zu werten ist, dass die Autoren neben klinischen Studien, die einen hohen Jadad-Score von 4 bis 5 Punkten aufweisen, auch Studien mit hohem Risiko für einen methodischen Bias nach den CochraneKriterien eingeschlossen haben, die immerhin 49\% der in den Metaanalysen der Autoren berücksichtigten klinischen Studien ausmachen. Leider konnte für die eingeschlossenen Studien nicht nachträglich geprüft werden, wie gut die Verblindung der Patienten tatsächlich funktioniert hat; auch ließen sich spezifische UAW nicht studienübergreifend analysieren. Mediatoren des Zusammenhangs zwischen Behandlung und therapeutischer Wirksamkeit lassen sich zudem mit aggregierten Daten nur mit eingeschränkter Sensitivität analysieren. Hier wären, wie die Autoren zu Recht anmerken, Metaanalysen auf der Basis individueller Patientendaten (IPDMA) eine interessante methodische Alternative gewesen. Deshalb sollten robuste Aussagen zum Einfluss von UAW auf die antidepressive Wirksamkeit von SSRI von den Ergebnissen einschlägiger IPDMA abhängig gemacht werden. Die vorliegende Publikation hat hierzu erste wichtige Ergebnisse vorgestellt, die über die Befunde einer älteren Metaanalyse [2] weit hinausgehen. Sollten sich die Resultate bestätigen, würden dadurch die bisherigen Empfehlungen, SSRI als pharmakologische Standardbehandlung depressiver Störungen einzusetzen, gestützt werden.

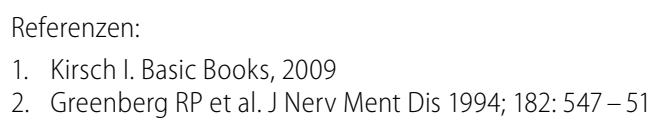

Dr. rer. biol. hum. Dipl.-Psych. Roland Mergl, Leipzig

Klinik und Poliklinik für Psychiatrie und Psychotherapie, Universitätsklinikum Leipzig AÖR

E-Mail: Roland.Mergl@medizin.uni-leipzig.de 